\title{
Distribución Vertical del Banco de Semillas de Euphorbia davidii Subils, en lotes Agrícolas de la Zona Centro de la Provincia de Buenos Aires, Argentina ${ }^{1}$
}

\author{
Vertical Distribution of Euphorbia davidii Subils Seedbank in Agricultural Lots in the Center of \\ Buenos Aires Province Argentina \\ NÚÑEZ FRÉ, F.R. ${ }^{2,4}$, JUAN, V.F. ${ }^{2}$ y CHANTRE, G. ${ }^{3}$
}

\begin{abstract}
RESUMEN - Euphorbia davidii Subils, es una maleza anual que está presente en la zona centro de la provincia de Buenos Aires, Argentina. Se realizaron estudios para determinar el banco de semillas de esta especie y su distribución vertical en lotes agrícolas de Azul y Olavarría. Se extrajeron 240 muestras de suelo, provenientes de 60 puntos distribuidos al azar, a cuatro niveles de profundidad: 0-5 cm, $5-10 \mathrm{~cm}, 10-15 \mathrm{~cm}$ y $15-20 \mathrm{~cm}$. Las extracciones se realizaron dos veces al año, en mayo y en septiembre. Las muestras fueron procesadas y las semillas separadas por tamizado en húmedo. Se evaluó germinación y viabilidad mediante

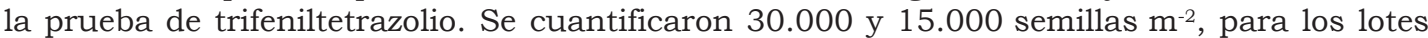
de Azul y Olavarría, respectivamente. En Olavarría, el 79\% de las semillas se encontraron de 0-5 cm, de profundidad, el 14\%, de 5-10 cm y el 7\% restante de $10-20 \mathrm{~cm}$. En Azul se observó una distribución vertical más homogénea. El $55 \%$ de las semillas se encontraron de $0-5 \mathrm{~cm}$ de profundidad, un $24 \%$ de $5-10 \mathrm{~cm}$, el $16 \%$ de $10-15 \mathrm{~cm}$ y el $5 \%$ en el muestreo más profundo. Las diferencias en la distribución se asociaron al manejo de los lotes. En ambas localidades la viabilidad de semillas, provenientes del estrato superficial, superó el $70 \%$. Las semillas de estratos superficiales recolectadas en el lote de Olavarria, inmediatamente después de la dehiscencia, presentaron dormición, en cambio en las semillas provenientes del lote de Azul para el mismo momento de muestreo, no se observó dormición.
\end{abstract}

Palabras-clave: lecherón, labranza, propágulos, viabilidad.

\begin{abstract}
Euphorbia davidii Subils a summer annual weed, that is present in the center of Buenos Aires province, Argentina. Field studies were conducted to determine the seed banks and the vertical distribution of this specie in agricultural lots, in Azul and Olavarria. Two hundred forty soil samples, from 60 randomly distributed points were extracted at four soil depths: 0-5, 5-10, 10-15 and 15-20 cm. Extractions were carried out twice a year, in May and September. Samples were processed and seeds separated by wet sieving. Germination and viability were assessed by triphenyltetrazolium test. Were quantified 30,000 and 15,000 seeds $\mathrm{m}^{-2}$ in Azul and Olavarria, respectively. In Olavarria, 79\% of the seeds were found of $0-5 \mathrm{~cm}$ depth, $14 \%$ of $5-10 \mathrm{~cm}$ and the remaining $7 \%$ of $10-20 \mathrm{~cm}$ depth. In Azul a more homogeneus vertical distribution was observed. The 55\% of seeds were found at 0-5 cm, 24\% depth; $24 \%$ at $5-10 \mathrm{~cm}, 16 \%$ at $10-15 \mathrm{~cm}$ and $5 \%$ in the deepest sampling. Differences in distribution were related to flock management. In both sites the viability of seeds from the surface layer exceeded 70\%. In Olavarria seeds collected of superficial layers immediately after dehiscence showed dormancy. In return, in seeds from Azul collected at the same time of sampling. Dormance was not be observed.
\end{abstract}

Keywords: david's spurge, tillage, propagules, viability.

Recebido para publicação em 23.4.2014 e aprovado em 16.7.2014.

2 Universidad Nacional del Centro de la Provincia de Buenos Aires, Azul, Provincia de Buenos Aires, República Argentina, <federicon@faa.unicen.edu.ar>; ${ }^{3}$ Universidad Nacional del Sur, Bahía Blanca, Provincia de Buenos Aires, República Argentina.

${ }^{4}$ Becario Comisión de Investigaciones Científicas de la Provincia de Buenos Aires (CIC-PBA). 


\section{INTRODUCCIÓN}

Euphorbia davidii Subils es una especie anual, de ciclo primavero-estival, originaria de Norteamérica, que inicialmente allí se difundió a través de las rutas. Posteriormente fue introducida en Sudamérica (Argentina) y Australia (Vladimirov \& Petrova, 2009).

En nuestro país ha sido registrada en las provincias de Córdoba y San Luis (Instituto de Botánica Darwinion, 2014). Últimamente ha sido reclasificada por Marchessi et al. (2011) para el centro y sur de la Provincia de Buenos Aires, a partir de ejemplares que anteriormente estaban registrados, desde la década del 80, como Euphorbia dentata Michaux (Juan et al., 1996).

En los Partidos de Azul y Olavarría se la menciona a partir del año 1983, asociada, principalmente, a cultivos de soja en los que se comporta como maleza muy competitiva localizada en machones (Juan et al., 1996). Actualmente se encuentra distribuida prácticamente en el $100 \%$ del área agrícola, en densidades que oscilan entre 300 a 900 plantas $\mathrm{m}^{-2}$ (Juan et al., 2011).

El banco de semillas puede ser definido como la reserva de semillas viables en el suelo, en profundidad o en la superficie (Roberts, 1981). En suelos agrícolas, éste constituye la principal fuente para la renovación de las poblaciones de plantas que infestan los cultivos (Cavers \& Benoit, 1989).

La germinación de semillas en el suelo, resulta del balance entre condiciones ambientales favorables y características intrínsecas de estos órganos de reproducción. Semillas viables y no dormidas, germinan con adecuados niveles de agua, oxígeno y temperatura, mientras que semillas enterradas pueden tener diferentes destinos, tales como pérdida de viabilidad, predación e inducción a dormición secundaria.

La dormición es uno de los principales mecanismos de preservación de especies en el banco de semillas, distribuyendo la germinación a lo largo del tiempo (Carmona, 1992).

En especies anuales, los individuos que emergen representan, solamente, una proporción de las semillas presentes en el suelo, pero, en general, existe una relación directa entre la abundancia de las especies en el lote y la abundancia de sus propágulos en el banco de semillas (Silvertown, 1982).

A nivel mundial el control químico es la principal estrategia en el control de malezas (Christoffoleti, 2004). Sin embargo, la necesidad de disminuir los costos de producción y la presión de la sociedad en contra del uso sistemático de herbicidas ha contribuido al desarrollo de una propuesta diferente, como el manejo integrado de malezas (Dessaint et al., 1996). Dentro del desarrollo de este sistema de manejo, el conocimiento de la dinámica del banco de semillas (composición, cantidad y distribución de semillas viables) es una etapa importante hacia el desarrollo de estrategias de control (Cardina \& Sparrow, 1996).

Debido a que existen escasos estudios de banco de semillas de las principales malezas, en la Argentina, y dado que E. davidii representa una de las principales especies invasoras de los lotes agrícolas de la región central de la provincia de Buenos Aires, en el presente trabajo se plantea como objetivo estudiar la abundancia, distribución vertical y viabilidad de las semillas de E. davidii en lotes donde se desarrolla agricultura continua, en la zona centro de la provincia de Buenos Aires, Argentina.

\section{MATERIALES Y MÉTODOS}

\section{Cuantificación y distribución vertical del banco de semillas de $E$. davidii}

Se realizaron estudios a campo en dos lotes agrícolas ubicados en el centro de la provincia de Buenos Aires, Argentina. Estos lotes fueron seleccionados por haber sido utilizados para agricultura en forma continua en el período (2000-2012) y por presentar una alta densidad de la maleza, que de acuerdo a los relevamientos previos superó las 500 plantas $\mathrm{m}^{-2}$ por año.

El lote de Olavarría se encuentra ubicado en la localidad de Sierras Bayas (240 m.s.n.m., 3658'0.99"; 60¹1’49.68"O) y el sistema de labranza utilizado durante la última década fue siembra directa continua; mientras que en el lote de Azul (148 m.s.n.m., 3650’8.75"S y 
5952'20.23"O) también bajo sistema de siembra directa, se realizó una labranza con disco en 2009.

El muestreo de cada lote consistió en la recolección de 240 muestras de suelo provenientes de 60 puntos distribuidos al azar, extraídas mediante un barreno. Las unidades de muestreo estuvieron representadas por cilindros de suelo de $8 \mathrm{~cm}$ de diámetro por $5 \mathrm{~cm}$ de alto, tomados a 4 niveles de profundidad: 0-5, 5-10, 10-15 y $15-20 \mathrm{~cm}$.

Las extracciones se realizaron dos veces al año; la primera durante el mes de mayo, luego de la dispersión de semillas, y la segunda en septiembre antes que comenzara la germinación en condiciones de campo.

Las muestras de suelo extraídas fueron almacenadas hasta su procesamiento a temperaturas menores a $15{ }^{\circ} \mathrm{C}$ y en oscuridad, para prevenir la germinación de las semillas.

La separación de la totalidad de los propágulos de las muestras de suelo se realizó en laboratorio, utilizando la técnica de tamizado en húmedo, que consistió en colocar las mismas sobre un tamiz malla 100, bajo un flujo de agua constante, para desagregar el suelo, identificar y juntar las semillas de la especie en estudio.

La cantidad de semillas proveniente de cada una de las dos extracciones anuales realizadas, en cada uno de los lotes relevados y de cada estrato de suelo, fueron registradas en forma independiente y los resultados fueron sometidos a análisis de varianza, en un diseño completamente al azar con cuatro repeticiones, las medias fueron separadas en base a la diferencia minima significativa (LSD) con un nivel de probabilidad $\mathrm{p}<0,05$; utilizando el software estadístico InfoStat versión 2012 (Grupo InfoStat, FCA, Universidad Nacional de Córdoba, Argentina).

\section{Evaluación de la germinación y viabilidad del banco de semillas}

A partir de las semillas obtenidas en la experiencia anterior, se realizaron ensayos para evaluar la germinación y la viabilidad.

Para esto, cuatro muestras de 30 semillas de cada localidad fueron dispuestas en placas de Petri sobre papel de filtro humedecido con agua destilada y colocadas en cámara de germinación a $18{ }^{\circ} \mathrm{C}$. Se realizaron recuentos del número de semillas germinadas cada 3 días, durante un período de 21 dias.

Las semillas que no germinaron, se sometieron al test de viabilidad, mediante la prueba 2,3,5-Trifenil tetrazolio (Perry, 1984), La viabilidad total de cada muestra se calculó en base a la cantidad de semillas germinadas en la primera evaluación, adicionando las que dieron resultado positivo de viabilidad en la prueba del tetrazolio.

Los datos de germinación y viabilidad se procesaron estadísticamente, en un diseño completamente al azar con cuatro repeticiones, las medias fueron separadas en base a la diferencia mínima significativa (LSD) con un nivel de probabilidad $\mathrm{p}<0,05$; utilizando el software estadístico InfoStat versión 2012 (Grupo InfoStat, FCA, Universidad Nacional de Córdoba, Argentina).

\section{RESULTADOS Y DISCUSIÓN}

En cuanto al total de propágulos cuantificados en cada sitio, se observó que en el lote ubicado en la localidad de Olavarría, con 10 años de siembra directa continua, se recolectaron más de 14.000 semillas $\mathrm{m}^{-2}$ de 0 a $20 \mathrm{~cm}$ en las dos épocas de muestreo, no detectándose diferencias significativas. En las muestras de suelo provenientes del lote localizado en Azul, que fue sometido a una labranza en 2009, se cuantificaron 28.000 semillas $\mathrm{m}^{-2}$ en el muestreo de mayo y 32.000 semillas $\mathrm{m}^{-2}$ en septiembre, registrándose diferencias significativas (Tabla 1).

En este último caso, teniendo en cuenta que la principal vía de dispersión de $E$. davidii es la dehiscencia elástica de los frutos y que los propágulos son relativamente grandes y carentes de órganos de dispersión anemócora, es poco probable que haya existido un aporte significativo de semilla de otros sitios. Posiblemente durante el primer muestreo de mayo, pudo haber habido un remanente de semillas en cápsulas sobre las plantas de E. davidii, o bien, algunas cápsulas cayeron al suelo sin abrirse y no fueron consideradas durante la separación. 
Tabla 1 - Cantidad total de semillas de $E$. davidii $\mathrm{m}^{-2}$ recolectadas sobre muestras de suelo de 0 a $20 \mathrm{~cm}$ de profundidad en dos lotes agrícolas de Olavarría y Azul, Provincia de Buenos Aires, Argentina

\begin{tabular}{|c|c|c|}
\hline Localidad & $\begin{array}{c}\text { Mayo } \\
\left(\text { semillas } \mathrm{m}^{-2}\right)\end{array}$ & $\begin{array}{c}\text { Septiembre } \\
\left(\text { semillas } \mathrm{m}^{-2}\right)\end{array}$ \\
\hline Olavarría & $14952 \mathrm{a}$ & $14692 \mathrm{a}$ \\
\hline Azul & $28081 \mathrm{~A}$ & $32678 \mathrm{~B}$ \\
\hline
\end{tabular}

La comparación es solo dentro de cada localidad.Los valores seguidos de una misma letra no difieren significativamente entre sí (test de Fisher, $\mathrm{p} \leq 0,05)$.

Por otro lado, si bien existe variación en la distribución horizontal del banco de semillas, es poco probable que este factor explique la diferencia observada, ya que en ambas fechas se tomaron 60 puntos de muestreo al azar en un mismo sector del lote y analizando las variaciones entre ambos muestreos por estrato, se aprecia que solamente existieron diferencias estadisticamente significativas en el estrato superior (Figura 1).

En estudios sobre banco de semilla total, realizados sobre un lote agrícola del partido de Azul en el año 2000, se encontró que la densidad total de semillas varió entre 20.000 y 60.000 semillas $\mathrm{m}^{-2}$. Ese estudio reflejó la existencia de una co-dominancia de tres especies más abundantes del banco de semillas Chenopodium album, Amaranthus quitensis y Digitaria sanguinalis, que representaron el $27,2 \%, 21,1 \%$ y $20,2 \%$ del banco de semillas, respectivamente; la única Euphorbiacea que se menciona de importancia es Euphorbia serpens (Requesens et al., 2004).

Si bien en el trabajo de referencia no se menciona la presencia de E. davidii, es importante destacar que nuestros estudios se realizaron en lotes bajo agricultura continua, con altas infestaciones de la maleza y en estos se encontró una cantidad de semillas de esta especie similar al banco total de semillas determinado en el estudio anterior. Por otro lado se destaca la evolución ocurrida en la composición florística de malezas de la región en la última década durante la cual $E$. davidii ha incrementado tanto su presencia como sus densidades (Juan et al., 2011) evidenciando claramente la capacidad invasiva y colonizadora de $E$. davidii.
El tradicional manejo de malezas, basado en el control químico, supone que existe un umbral, que es definido como la densidad de malezas en la que el costo del control es equivalente al valor del rendimiento del cultivo salvado por ese control (Cousens, 1987). Debido a este razonamiento, habrá densidades de malezas que no afectarian el rendimiento del cultivo en forma significativa como para justificar la aplicación de un herbicida, desde el punto de vista económico; por lo tanto cierto nivel de densidad de malezas permanecerian en el campo, y llevarian a un incremento en la magnitud del banco de semillas (Hartzler, 1996).

Un incremento en el tamaño del banco de semillas dificultará los programas de manejo de malezas, debido a que una alta densidad inicial puede llevar a que exista un elevado remanente de individuos, aún luego de la aplicación de un control químico con una efectividad superior 85\% (Taylor \& Hartzler, 2000), llevando a la necesidad de recurrir a un nuevo tratamiento $\mathrm{u}$ otra alternativa de control.

En la Figura 1 se presentan los resultados de la distribución vertical de las semillas en el lote de la localidad de Olavarría. Para el primer muestreo realizado en mayo, se observó que más del $80 \%$ de los propágulos se encontraron en los primeros $5 \mathrm{~cm}$ del suelo, lo cual resulta previsible por la forma de dispersión de las semillas y la reciente dehiscencia de las cápsulas que, en general,

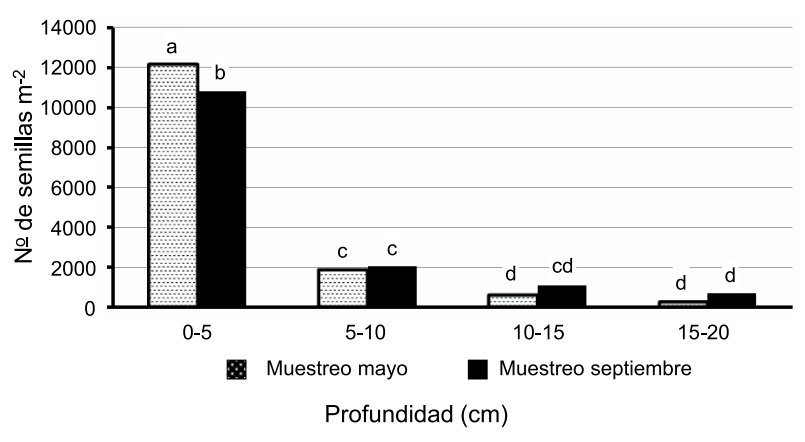

Letras iguales indican que no existen diferencias significativas entre sí (test de Fisher $\mathrm{p} \leq 0,05$ ).

Figura 1 - Cantidad de semillas de E. davidii $\mathrm{m}^{-2}$ recolectadas en 4 estratos de profundidad de suelo $(0-5,5-10,10-15$ y $15-$ $20 \mathrm{~cm}$ ) en muestras extraídas en mayo y septiembre en Olavarría. 
se produce durante los meses de marzo y abril. En este sentido, en relevamientos realizados en siembra directa para diversas malezas, se encontró que entre el 60 y 99\% de las semillas se ubicaban a profundidades de $0-5 \mathrm{~cm}$ (Ghersa \& Martínez-Ghersa, 2000).

En el segundo estrato muestreado $(5-10 \mathrm{~cm})$ se cuantificaron menos de 2.000 semillas $\mathrm{m}^{-2}$, que representó alrededor del 13\% del total y, el $7 \%$ restante se encontró en los dos estratos más profundos entre los 10 y $20 \mathrm{~cm}$ de suelo muestreados.

En el muestreo realizado a los 5 meses de producida la dehiscencia (septiembre), se obtuvieron resultados similares, no obstante, se observó una variación significativa en la cantidad de semillas del primer estrato, que registró una disminución de, aproximadamente, un $8 \%$ respecto de la cantidad registrada en mayo y, a su vez se observó un incremento, estadísticamente no significativo, entre el 1 y el $4 \%$ en los tres estratos establecidos entre los 5 y los $20 \mathrm{~cm}$ de profundidad. La reducción en el número de propágulos del estrato superficial, puede ser atribuida al enterrado de las semillas por el arrastre que ocasiona el agua, a medida que ingresa en el suelo luego de las lluvias, como así también a la actividad de la macrofauna del suelo, incluyendo a las hormigas.

Es importante destacar que en el caso de siembra directa continua, la mayor proporción de las semillas presentes cerca de la superficie, sufrirán un mayor riesgo de predación, envejecerán más rápidamente y presentarán tasas de mortalidad incrementadas por germinación en ambientes desfavorables (Ballaré et al., 1988).

Por otra parte, en periodos de sequía, las grandes grietas formadas en el suelo podrian permitir a las semillas caer en capas más profundas del suelo (Ghersa \& MartinezGhersa, 2000). Este fenómeno es posible que explique en parte la disminución de la cantidad de semillas en el estrato superficial y la tendencia a un incremento en las capas más profundas.

La disminución en el número de semillas entre ambos muestreos, también puede deberse en parte al transporte secundario mediado por hormigas. Existen numerosos trabajos donde se menciona la interacción entre semillas de especies de la familia de las Euphorbiaceas y hormigas. Dentro de los principales factores bióticos de desaparición de semillas de Euphorbiaceas (incluyendo roedores y aves), las hormigas son ampliamente el factor más influyente. Esa desaparición ocurre tanto por transporte secundario hasta los hormigueros, como por predación de las mismas (Espadaler \& Gómez, 1996).

La presencia de un elaiosoma, es un factor importante en la interacción hormiga-semilla; dado que aquellas semillas que cuentan con el mismo, tienen una probabilidad siete veces mayor de ser transportadas a los hormigueros por hormigas no predadoras, esto sustenta la teoría de que la mirmecocoria, es una estrategia para evitar predadores de semillas (Espadaler \& Gómez, 1997).

En la Figura 2 se presentan los resultados de la distribución de semillas por estrato obtenida para el lote ubicado en la localidad de Azul. Como ya fue destacado, en este lote el número total de propágulos resultó mayor que en el caso de Olavarría, representando aproximadamente el doble (Tabla 1). En cuanto a la distribución se observa que independientemente del momento de muestreo en los primeros $5 \mathrm{~cm}$ de suelo se encontraron el $55 \%$ de los propágulos con respecto al total relevado, más de un $20 \%$ se ubicó entre los 5 y $10 \mathrm{~cm}$ de profundidad, aproximadamente el $15 \%$ en el estrato de 10 a $15 \mathrm{~cm}$ y alrededor de un $5 \%$ entre los 15 y $20 \mathrm{~cm}$ de suelo muestreado (Figura 2 y Tabla 2).

De la comparación de las dos fechas de muestreo se observa que solamente existieron diferencias significativas en el número de semillas para el primer estrato de 0 a $5 \mathrm{~cm}$, que de igual forma que en el número total de propágulos presentado en la Tabla 1, registró un incremento significativo en la cantidad de semillas en el muestreo de septiembre con respecto al relevamiento previo de mayo.

Como se había mencionado anteriormente, esta diferencia puede explicarse en primer lugar, debido a un remanente de semillas en cápsulas sobre las plantas de E. davidii, o por cápsulas que cayeron al suelo conteniendo semillas. Otro factor a considerar, podría ser una menor predación de semillas, 


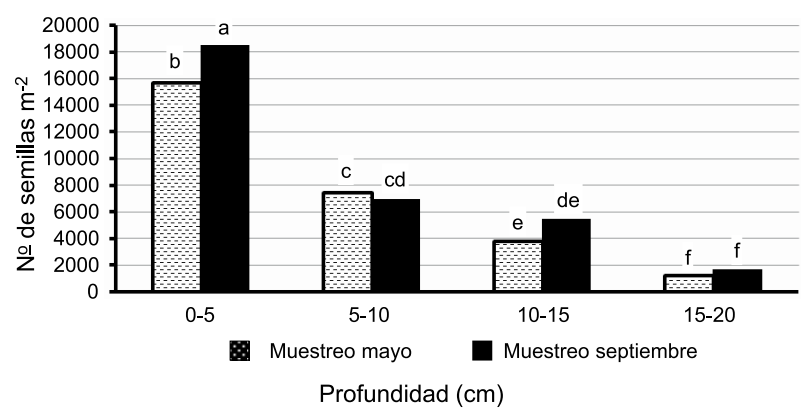

Letras iguales indican que no existen diferencias significativas entre sí (test de Fisher, $\mathrm{p} \leq 0,05$ ).

Figura 2 - Cantidad de semillas de E. davidii $\mathrm{m}^{-2}$ recolectadas en 4 estratos de profundidad de suelo (0-5, 5-10, $10-15$ y $15-20 \mathrm{~cm})$ en muestras extraídas en mayo y septiembre en Azul.

Tabla 2 - Distribución vertical de las semillas de E. davidii encontradas hasta los $20 \mathrm{~cm}$ de profundidad, en porcentaje respecto del total cuantificado, para los dos sitios muestreados en mayo y septiembre

\begin{tabular}{|c|c|c|c|c|}
\hline \multirow{2}{*}{ Profundidad } & \multicolumn{2}{|l|}{ Mayo } & \multicolumn{2}{l|}{ Septiembre } \\
\cline { 2 - 5 } & Olavarría & Azul & Olavarría & Azul \\
\hline $0-5$ & $81,02 \mathrm{a}$ & $55,42 \mathrm{~b}$ & $72,60 \mathrm{a}$ & $55,15 \mathrm{~b}$ \\
\hline $5-10$ & $13,02 \mathrm{~d}$ & $26,15 \mathrm{c}$ & $14,64 \mathrm{~d}$ & $22,13 \mathrm{c}$ \\
\hline $10-15$ & $3,94 \mathrm{e}$ & $14,13 \mathrm{~d}$ & $7,40 \mathrm{e}$ & $17,12 \mathrm{~d}$ \\
\hline $15-20$ & $2,00 \mathrm{e}$ & $4,02 \mathrm{e}$ & $5,36 \mathrm{e}$ & $5,60 \mathrm{e}$ \\
\hline LSD 0,05 & \multicolumn{2}{|c}{9.49} & \multicolumn{2}{|c}{8.38} \\
\hline
\end{tabular}

La comparación se realiza dentro de cada momento de muestreo. Los valores seguidos de una misma letra no difieren significativamente entre sí (test de Fisher, $\mathrm{p} \leq 0,05$ ).

que usualmente ocurre en sitios donde hay disturbios como labranzas (Ballaré et al., 1988). Sumado a este efecto de menor predación, podria haber ocurrido un menor transporte por parte de las hormigas, por destrucción de hormigueros durante la labranza. Dentro de los factores bióticos que podrian influir, también se podría destacar el aporte de semillas desde otros sitios que hayan sido consumidas por aves y eliminadas junto con el guano. Existen diversos trabajos en donde se ha evaluado la dispersión de semillas de Euphorbiaceas por diferentes especies de aves (Blockstein et al., 1987; Wald et al., 2005) en los cuales se puede apreciar que este factor, si bien puede contribuir al proceso de dispersión, es de una importancia secundaria.

Otro factor que resta considerar, es el arrastre de semillas de $E$. davidii sobre el suelo luego de una lluvia. Al tratarse de semillas de un tamaño de $2-2,5 \mathrm{~mm}$, es probable que puedan ser arrastradas por una lluvia de moderada intensidad; con lo cual se daría una dispersión secundaria de semillas, y estas podrian incorporarse a las posiciones más bajas del paisaje.

Comparando la distribución vertical para los dos sitios en estudio, en la Tabla 2 se presentan los datos en porcentaje respecto al total de semillas encontradas.

Se puede observar la influencia de la labranza realizada en el lote de Azul, sobre la distribución vertical de las semillas en el perfil.

En el sitio ubicado en Olavarría, con una década de agricultura en siembra directa, la mayor parte de las semillas está posicionada superficialmente. Del total de semillas recolectadas en este lote, alrededor de 14.000 propágulos $\mathrm{m}^{-2}$, más de 10.000 se encontraron en los primeros $5 \mathrm{~cm}$ del suelo, mientras que en Azul más de la mitad de los 30.000 propágulos $\mathrm{m}^{-2}$ extraídos, se encontraron en este estrato, independiente del momento del muestreo. Esta tendencia, también, se manifestó en profundidades de 5 a $10 \mathrm{~cm}$ y de 10 a $15 \mathrm{~cm}$, donde resulta notorio el impacto de la labranza realizada durante el último trieño agrícola.

Carter \& Ivany (2006), detectaron diferencias en la distribución de las semillas de malezas en el suelo y encontraron interacción entre el sistema de labranza y el tamaño de la semilla. En este trabajo, donde se evaluaron sistemas de labranza profunda, superficial y siembra directa, se observaron cambios más notorios en las especies de semillas más pequeñas como Gnaphalium uliginosum $(0,5 \mathrm{~mm})$ y Chenopodium album $(1 \mathrm{~mm})$, en contraste con propágulos de Ranunculus repens ( $3 \mathrm{~mm})$. Esto se corresponde con los estudios realizados por Clements et al. (1996), quienes sugieren que el efecto de la labranza es dependiente de la especie de la maleza y está relacionado principalmente con el tamaño de la semilla.

En nuestro trabajo encontramos diferencias en la distribución de las semillas en el suelo, luego de un solo año de labranza, incluso 
considerando que el tamaño de las semillas de $E$. davidii es relativamente grande.

Comparando labranzas minimas y tradicionales, Ekelme et al. (2005), encontraron que para dos Euphorbiaceas (Euphorbia hyssopifolia, y Euphorbia heterophylla), el número de propágulos en el suelo no resultó afectado por el sistema de labranza utilizado.

La distribución de las semillas en los diferentes estratos observada en Azul, donde el suelo fue laboreado, puede ser explicada en términos de probabilidad de movimiento de semillas a lo largo del perfil del suelo. Según los estudios realizados por Mohler et al. (2006), la probabilidad de que después de una labranza las semillas superficiales se ubiquen a más de $10 \mathrm{~cm}$ es superior al 70\%, mientras que la probabilidad de traer a la superficie semillas que se encontraban inicialmente a $14-18 \mathrm{~cm}$, es de sólo un $40 \%$. De esto se deduce, que una labranza profunda tiene un efecto neto de transporte de semillas superficiales a profundidades mayores a $10 \mathrm{~cm}$.

Evaluando la respuesta de la germinación en las semillas de la localidad de Olavarría, se encontraron diferencias entre los dos momentos de muestreo evaluados (Figura 3).

Las semillas extraídas en mayo, de los dos estratos superficiales $(0-5$ y $5-10 \mathrm{~cm})$, presentaron un bajo porcentaje de germinación $(<10 \%)$, mientras que las muestras más profundas superaron el $50 \%$ de germinación y no se diferenciaron estadísticamente entre

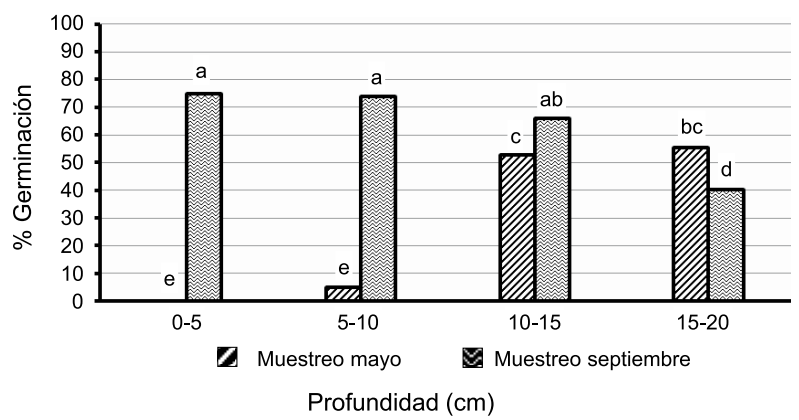

Letras iguales indican que no existen diferencias significativas entre sí (test de Fisher, $\mathrm{p} \leq 0,05$ ).

Figura 3 - Porcentaje de germinación, en laboratorio, de semillas de E. davidii $\mathrm{m}^{-2}$ extraídas de 4 estratos de profundidad en un lote agrícola de Olavarría, en mayo y septiembre. sí. Las semillas extraídas en septiembre desde 0 hasta los $15 \mathrm{~cm}$ de profundidad no presentaron diferencias significativas, obteniéndose una germinación entre el $65 \%$ y el $75 \%$, diferenciándose de la muestra más profunda $(15-20 \mathrm{~cm})$ que logró una germinación de alrededor del 40\%.

Estos cambios en el porcentaje de germinación pueden deberse a los propios ciclos de dormición de las semillas, como así también por diferentes factores que influyen en la germinación: intensidad lumínica, fotoperiodo, luz, temperatura y sus fluctuaciones, nitratos, niveles de $\mathrm{O}_{2}, \mathrm{CO}_{2}, \mathrm{pH}$, humedad y abrasión física de la cubierta seminal son algunos de ellos (Silvertown, 1982).

La temperatura es un requisito importante para la superación de la latencia y puede interferir en los flujos anuales de infestación de malezas (Benech-Arnold et al., 1988). En el banco de semillas del suelo la temperatura es el principal responsable para la latencia de las semillas de malezas, en las regiones de clima templado en las que existen fluctuaciones diurnas y estacionales, que ayudan a romper la latencia o reinducir a la dormición según la especie (Forcella, 1998).

Al analizar la viabilidad de las semillas evaluadas, se observó que, a pesar de los bajos porcentajes de germinación obtenidos en la extracción de mayo en los estratos de 0-5 y $5-10 \mathrm{~cm}$, la viabilidad de estos superó el $70 \%$ llegando a valores similares a los de las muestras extraídas en septiembre (Figura 4).

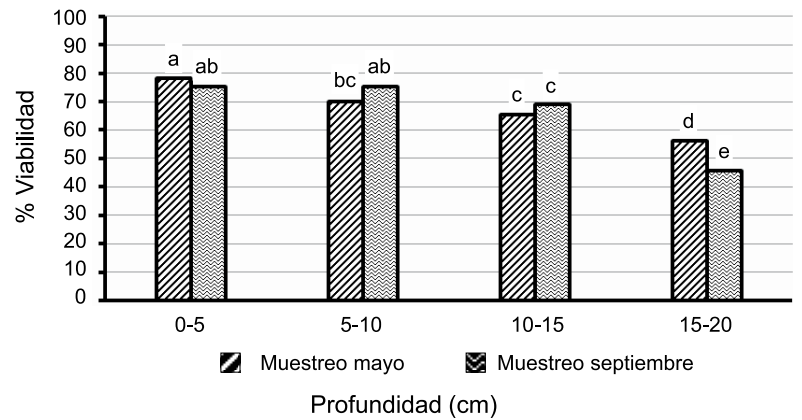

Letras iguales indican que no existen diferencias significativas entre sí (test de Fisher, $\mathrm{p} \leq 0,05$ ).

Figura 4 - Porcentaje de viabilidad de semillas de E. davidii m${ }^{2}$ extraídas de 4 estratos de profundidad de suelo de un lote agrícola de Olavarría, en mayo y septiembre. 
Por otra parte, se evidencia una clara diferencia en el comportamiento de los propágulos encontrados en el banco de semillas superficial (muestras de $0-10 \mathrm{~cm}$ ) que, posiblemente, están mayoritariamente conformados por la dispersión de semillas del año, respecto al resto del banco existente a mayores profundidades, compuesto por semillas que, seguramente, llevan varios años en el suelo.

También es posible observar que entre los momentos de extracción de la muestra sólo se encontraron diferencias en la viabilidad en el estrato más profundo.

Por otra parte, evaluando dentro de una misma fecha de muestreo, se encontraron disminuciones estadisticamente significativas de la viabilidad, a medida que se incrementa la profundidad de muestreo, lo cual es lógico, si se supone que las semillas más profundas son cronológicamente anteriores que las más superficiales.

Sobre las muestras de suelo recolectadas en el lote de Azul, en el muestreo de mayo, el mayor porcentaje de germinación $(70 \%)$ se obtuvo a partir de las semillas extraídas de 0 a $5 \mathrm{~cm}$, aunque este valor no se diferenció estadísticamente de los propágulos enterrados entre los 15 y $20 \mathrm{~cm}$ que alcanzaron un $50 \%$ de germinación (Figura 5).

En las semillas provenientes de las muestras de suelo recolectadas en septiembre, al igual que en el caso anterior, las semillas superficiales $(0-5 \mathrm{~cm})$ obtuvieron el $70 \%$ de germinación, diferenciándose estadísticamente del resto de las profundidades.

Con respecto a la evaluación de la viabilidad, en las muestras de mayo y septiembre los mayores valores que superaron el $70 \%$ se obtuvieron en el estrato superficial, que se diferenció del resto de las profundidades, cuyos valores estuvieron entre 50 y $60 \%$ para este parámetro (Figura 6).

Comparando la información obtenida en la germinación y viabilidad, en primer lugar, se puede apreciar que prácticamente todas las semillas viables del estrato superior germinaron. En el resto de los estratos no todas las semillas viables germinaron en el periodo de evaluación.

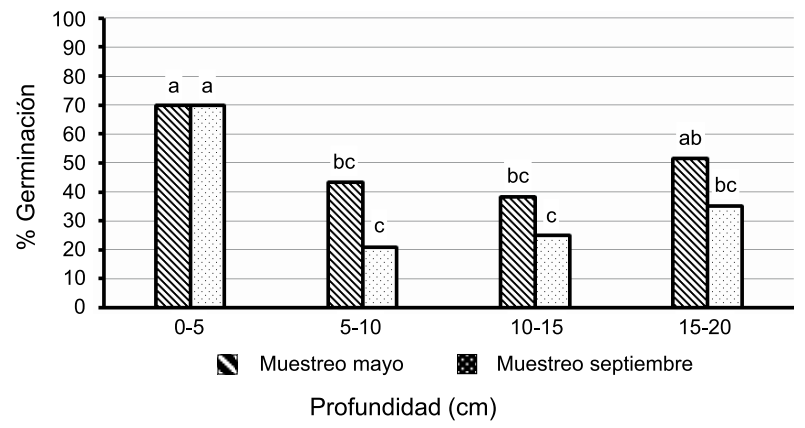

Letras iguales indican que no existen diferencias significativas entre sí (test de Fisher, $\mathrm{p} \leq 0,05$ ).

Figura 5 - Porcentaje de germinación, en laboratorio de semillas de E. davidii $\mathrm{m}^{-2}$ extraídas de 4 estratos de profundidad de suelo de un lote agrícola de Azul, en mayo y septiembre.

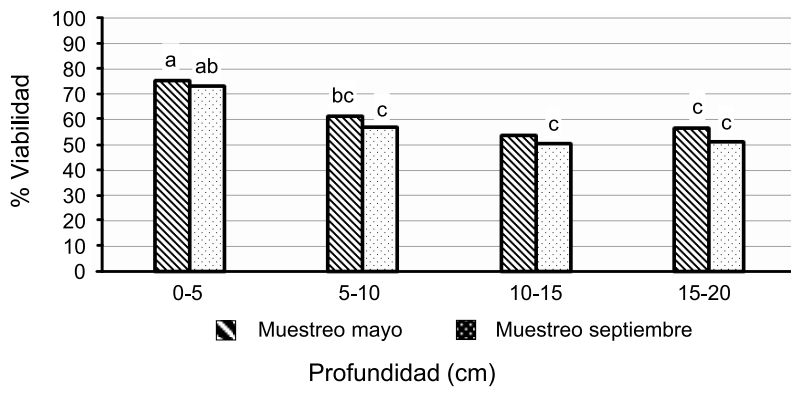

Letras iguales indican que no existen diferencias significativas entre sí (test de Fisher para $\mathrm{p} \leq 0,05$ ).

Figura 6 - Porcentaje de viabilidad de semillas de E. davidii $\mathrm{m}^{-2}$ extraídas de 4 estratos de profundidad de suelo de un lote agrícola de Azul, en mayo y septiembre.

La labranza podría tener diferentes efectos sobre la viabilidad y persistencia del banco de semillas. Cuando las labranzas son poco frecuentes, pero profundas, hay una alta probabilidad de que las semillas se muevan a capas profundas, donde la germinación es muy baja. Si la supervivencia es baja en esa profundidad, la tendencia es hacia una declinación del número de semillas en el banco; ya que morirán antes de ser llevadas a la superficie por otro laboreo. Por el contrario, si la supervivencia es alta en profundidad, los laboreos tendrán un efecto beneficioso, ya que moverán las semillas hacia sitios seguros. Por lo cual, la dinámica de una población de malezas con banco de semillas persistente, estará influenciado por la frecuencia e intensidad de laboreos (Eager et al., 2013). En este mismo 
sentido, estudios realizados durante 72 meses indican que la longevidad de las semillas está relacionada en forma directa con el aumento de profundidad y en forma inversa con la intensidad de remoción (Leguizamón, 1983).

Con respecto a la longevidad de semillas de malezas en un suelo cultivado, Barralis et al., (1988) considera dos grupos de especies: aquellas con una alta de declinación anual en el número de semillas en el banco, dentro de las que se encuentran Alopecurus myosuroides, Avena fatua, Centaurea cyanus, Galium aparine, Lapsana communis y Matricaria perforat; y especies con una baja tasa de disminución anual, dentro de las cuales se incluyen algunas Euphorbiaceas, como Euphorbia exigua. Roberts y Dawkins (1967), mencionan que eliminando el aporte de nuevos propágulos, la reducción en el número de semillas viables del banco, fue de un $22 \%$ anual para sitios no disturbados y de un 30 a 36\% para situaciones con dos y cuatro disturbios por año, respectivamente.

En trabajos realizados con el fin de determinar la viabilidad y el rango de temperaturas óptimas de germinación, para semillas de Euphorbia davidii provenientes de dos cosechas (1995 y 2009), se determinó que, en general, la viabilidad de semillas recién cosechadas supera el 90\%. En cuanto a la germinación para semillas provenientes de la cosecha 1995, conservadas durante 14 años, en condiciones de laboratorio, se obtuvieron valores de germinación de un 40\%, mientras que para propágulos recolectados en 2009 la germinación fue del 73\% (Marchessi et al., 2011). Si bien no conocemos con exactitud la longevidad de las semillas E. davidii en el suelo, es posible que la misma sea similar a otras Euphorbiaceas, con lo cual es necesario pensar el control de esta maleza, como una problemática a mediano o largo plazo.

De todas formas, es interesante destacar que en ambas localidades se encontraron evidencias de comportamientos diferenciales entre el estrato superior y el resto del banco de semillas. Las semillas del estrato $0-5 \mathrm{~cm}$, provienen, principalmente, del último aporte de semillas de la planta madre, y, por lo tanto, su comportamiento y biotipos, se van a corresponder con las condiciones de crecimiento de
E. davidii del ciclo inmediatamente anterior. El resto del banco de semillas está principalmente conformado por propágulos de diferentes edades, que provienen de plantas desarrolladas en diversas condiciones ambientales y compuesto por distintas proporciones de genotipos, con lo cual sería probable un comportamiento diferente al del estrato superficial. Esta variabilidad en la composición del total del banco de semillas, brinda un efecto "buffer", que le permite a la maleza contar con un pool genético amplio, que favorece la adaptación evolutiva a la variabilidad ambiental de la región (Levin, 1990).

Evidentemente la diferencia en la germinación del banco superficial entre las extracciones realizadas en mayo para las dos localidades, indican que el fenómeno de dormición se encuentra posiblemente en relación al efecto materno, y la interacción genotipo por ambiente, aspectos que serian materia de futuros estudios.

\section{LITERATURA CITADA}

BALLARÉ, C. L. et al. The fate of Datura ferox L. seeds in the soil as affected bycultivation depth of burial and degree of maturity. Ann. Appl. Biol., v. 112, n. 1, p. 337-345, 1988.

BARRALIS, G. et al. Longevity of annual weed seeds in a cultivated soil. Weed Res., v. 28, n. 6, p. 407-418, 1988.

BENECH-ARNOLD, R. L. et al.. The role of fluctuating temperatures in the germination and establishment of Sorghum halepense (L.) Pers. Regulation of germination under leaf canopies Func. Ecol., v. 2, n. 3, p. 311-318, 1988.

BLOCKSTEIN, D. et al. Dispersal of leafy spurge seeds (Euphorbia esula) by Mourning Doves (Zenaidura macroura). Weed Sci., v. 35, n. 2, p. 160-162, 1987.

CARDINA, J.; SPARROW, D. A comparison of methods to predict weed seedling populations from the soil sedbank. Weed Sci., v. 44, n. 1, p. 46-51, 1996.

CARMONA, R. Problemática e manejo de bancos de sementes de invasoras em solos agrícolas. Planta Daninha, v. 10, n. 12, p. 5-16, 1992.

CARTER, M. R.; IVANY, J. A. Weed seed bank composition under three long-term tillage regimes on a fine sandy loam in Atlantic Canada. Soil Tillage Res., v. 90, n. 1-2, p. 29-38, 2006.

Planta Daninha, Viçosa-MG, v. 32, n. 4, p. 709-718, 2014 
CAVERS, P. B.; BENOIT, D. L. Seed banks in arable land. In: LECK, M. A; PARKER, V. T.; SIMPSON, R. L. Ecology of soil seed banks. San Diego: Academic Press, 1989. p. 309328 .

CHRISTOFFOLETI, P. J. Aspectos de resistência de plantas daninhas a herbicidas. Campinas: HRAC-BR, 2004. $100 \mathrm{p}$

CLEMENTS, D. R. et al. Tillage effect on weed seed return and seedbank composition. Weed Sci., v. 44, n. 2, p. 314-322, 1996.

COUSENS, R. Theory and reality of weed control thresholds. Plant Protec. Quart., v. 2, n. 1, p. 13-20, 1987.

DESSAINT, F. et al. Precision of soil seedbank sampling: how many soil cores? Weed Res., v. 36, n. 1, p. 143-151, 1996.

EAGER, E. A. et al. Disturbance frequency and vertical distribution of seeds affect long-term population dynamics: A mechanistic seed bank model. Am. Natur., v. 182, n. 2, p. 180-190, 2013.

EKELEME, F. et al. Weed seedbank response to planted fallow and tillage in southwest Nigeria. Agrofor. Syst., v. 63, n. 3, p. 299-306, 2005.

ESPADALER, X.; GÓMEZ, C. Seed production, predation and dispersal in the Mediterranean myrmecochore Euphorbia characias (Euphorbiaceae). Ecography, v. 19, n. 1, p. 7-15, 1996

ESPADALER, X.; GÓMEZ, C. Soil surface scanning and transport of Euphorbia characias seeds by ants. Acta Oecologica, v. 18, n. 1, p. 39-46. 1997.

FORCELLA, F. Real-time assessment of seed dormancy and seedling growth for weed management. Seed Sci. Res., v. 8, n. 2, p. 201-209, 1998.

GHERSA, C. M.; MARTIiNEZ-GHERSA, M. A Ecological correlates of weed seed size and persistence in the soil under different tilling systems: implications for weed management. Field Crops Res., v. 67, n. 2, p. 141-148, 2000.

HARTZLER, R. G. Velvetleaf (Abutilon theophrasti) population dynamics following a single year's seed rain. Weed Technol., v. 10, n. 3, p. 581-586, 1996.

INSTITUTO DE BOTÁNICA DARWINION (on line). Flora del Conosur. Catálogo de Plantas Vasculares. 2014. Disponible en: < http://www.darwin.edu.ar/Proyectos/ FloraArgentina/FA.asp>. Consultado el: 10 fev. 2014.
JUAN, V. F. et al. Estudios sobre Lecherón (Euphorbia dentata Michaux) en la zona centro de la provincia de Buenos Aires. Planta Daninha, v. 14, n. 2, p. 102-109, 1996.

JUAN, V. F. et al. Control de Euphorbia davidii (Lecherón) con Glifosato. R. Esp. Malezas - Aapresid, 2011. 10 p.

LEGUIZAMÓN, E. S. Dinámica poblacional de sorgo de alepo (Sorghum halepense L. Pers) en soja. Enfoque del estudio. Primeras estimaciones del impacto de diferentes niveles de control en el banco de propágulos. Oliveros: Estación Experimental Agropecuaria - INTA, 1983. 12 p. (Informe Técnico, 32)

LEVIN, D. The seed bank as a source of genetic novelty in plants. Am. Natur., v. 135, n. 4, p. 563-572, 1990.

MARCHESSI, J. E. et al. Presencia de Euphorbia davidii Subils (Euphorbiaceae) en la Provincia de Buenos Aires: Morfología y Anatomía de la espécie. Kurtziana, v. 36, n.1, p. 45-53, 2011.

MOHLER, C. L. et al. Vertical movement of weed seed surrogates by tillage implements and natural processes. Soil Tillage Res., v. 86, n. 1, p. 110-122, 2006.

PERRY, D. A. Manual de métodos de ensayos de vigor. Publicación Española. Instituto Nacional de semillas y Plantas de Vivero. 1984. p. 45-51.

REQUESENS, E. et al. Banco de semillas de malezas a lo largo de un gradiente microtopográfico en un suelo agrícola de Azul (Buenos Aires). Ecol. Austral, v. 14, n. 2. p. 141-147, 2004.

ROBERTS, H. A. Seed banks in the soil. Adv. Appl. Biol., v. 6, n. 1, p. $1-55,1981$

ROBERTS, H. A.; DAWKINS, P. A. Effect of cultivation on the number of viable weed sedes in soil. Weed Res., v. 7, n. 1, p. 290-301, 1967.

SILVERTOWN, J. W. Introduction to plant population ecology. London. Longman Group, 1982. 209 p.

TAYLOR, K.; HARTZLER, R. Effect of seed bank augmentation on herbicide efficacy. Weed Technol., v. 14, n. 2, p. 261-267, 2000.

VLADIMIROV, V.; PETROVA, A. A new alien species of Euphorbia (Euphorbiaceae) to the Bulgarian flora. Phytol. Balcanica, v. 15, n. 3, p. 343-345, 2009.

WALD, J. et al. Dispersal of Leafy Spurge (Euphorbia esula L.) Seeds in the Feces of Wildlife. Am. Midland Natur., v. 154 , n. 2, p. $342-357,2005$. 